\title{
Combining Multiple Forms of Evidence While Filtering
}

\author{
Yi Zhang * Jamie Callan \\ Information System and Technology Management Language Technologies Institute \\ School of Engineering School of Computer Science \\ University of California, Santa Cruz \\ Santa Cruz, CA 95064, USA \\ Carnegie Mellon University \\ Pittsburgh, PA 15213, USA \\ yiz@soe.ucsc.edu \\ callan@cs. cmu . edu
}

\begin{abstract}
This paper studies how to go beyond relevance and enable a filtering system to learn more interesting and detailed data driven user models from multiple forms of evidence. We carry out a user study using a real time web based personal news filtering system, and collect extensive multiple forms of evidence, including explicit and implicit user feedback. We explore the graphical modeling approach to combine these forms of evidence. To test whether the approach can help us understand the domain better, we use graph structure learning algorithm to derive the causal relationships between different forms of evidence. To test whether the approach can help the system improve the performance, we use the graphical inference algorithms to predict whether a user likes a document based on multiple forms of evidence. The results show that combining multiple forms of evidence using graphical models can help us better understand the filtering problem, improve filtering system performance, and handle various data missing situations naturally.
\end{abstract}

\section{Introduction}

An adaptive personal information filtering system is an autonomous agent that delivers information to the user in a dynamic environment over a period of time. A common filtering approach is adapting existing text classification/retrieval algorithms to classify incoming documents as either relevant or non relevant using user profiles learned from explicit user feedback on documents the user has seen. However, there are other important criteria for the user besides relevance, such as readability (Collins-Thompson and Callan, 2004), novelty (Harman, 2003), and authority (Kleinberg, 1998). Besides, much information about the user and the document can be collected by a filtering system. These suggest a way to improve the current filtering system: going beyond relevance and using multiple forms of evidence.

This research was done while at the Language Technologies Institute, Carnegie Mellon University.
Unfortunately, there is no standard evaluation data set for this research, and there is not much work on finding a good theory to combine various forms of evidence. To solve the first problem, we designed a user study and collect thousands of cases with multiple forms of evidence, including the content of a document, explicit and implicit user feedback, such as a user's mouse usage, key board usage, document length, novelty, relevance, readability, authority, user profile characteristics, news source information, and whether a user likes a document or not. Solving the second problem is very challenging. A good model should have the representation power to combine multiple forms of evidence; it should be able to help us understand the relationships between various forms of evidence; it should use the evidence to improve filtering system performance; and it should handle various problems like missing data in an operational environment robustly.

On the other hand, researchers have identified three major advantages of graphical modeling approach: 1) it provides inference tools to naturally handle situations of missing data entry because of the conditional dependencies encoded in the graph structure; 2) it can learn causal relationships in the domain, thus help us to understand the problem and to predict the consequences of intervention; and 3) it can easily combine prior knowledge (such as partial information about the causal relationship) with data in this framework. This approach has been applied to model computer software users (Horvitz et al., 1998), car drivers (Pynadath and Wellman, 1995), and students (Conati et al., 1997). Motivated by the prior work, we choose to use graphical models as our solution. To understand relationships between various forms of evidence, we use the causal graph structure learning algorithms (advantage 2), together with some prior knowledge of the domain (advantage 3), to derive the causal relationships between different user feedback, actions and user context. To improve the existing filtering system, especially in the situation of missing data, we use statistical inference tools to predict how a user will like a document, using information available in different missing evidence situations (advantage 1). We also try linear regression as an alternative approach.

The following sections describe our efforts towards 
Figure 1: The user study system structure. The structured information, such as user feedback and crawler statistics, are kept in the database. The content of each web page crawled is saved in the news repository.

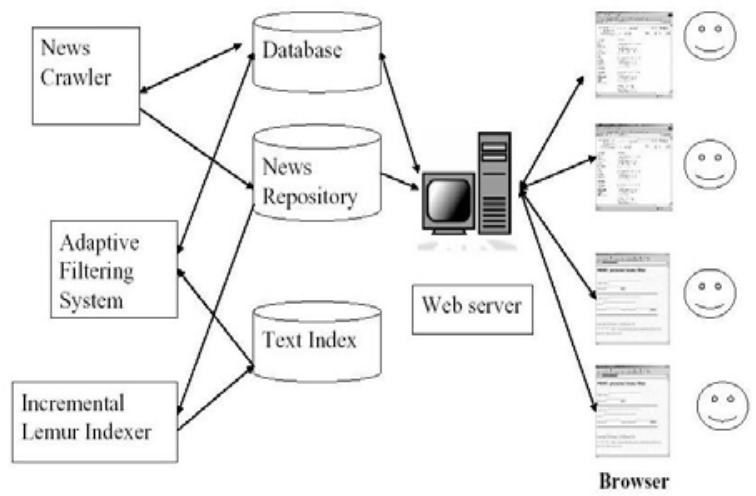

collecting data and customizing the graphical modeling approach to combine multiple forms of evidence for filtering. We begin with a description of the user study in Section 2, followed by some preliminary data analysis on the data collected in Section 3. Section 4 explores causal structure learning algorithm to understand the relationships between various forms of evidence from the data and Section 5 explores how to improve the system performance using multiple forms of evidence. Section 6 discusses related work and how this work differs from existing work, and Section 7 concludes.

\section{User Study}

No existing filtering database contains the level of detail that we needed for our study, so we developed a web based news story filtering system to collect an evaluation data set (Figure 1). This system constantly gathers and recommends information to the users. The system includes a crawler with 8000 candidate RSS news feeds (Pilgrim, 2002) to crawl every day. The Lemur indexer indexes the crawled document stream incrementally, and an adaptive filtering system recommends documents to the users using a modified logistic regression algorithm (Zhang, 2004). Users read and evaluate what the system has delivered to them. An example of the web interface after user login is in Figure 2.

More than 20 paid subjects from 19 different programs at Carnegie Mellon University, who are otherwise not affiliated with our research, participated in the study for 4 weeks. We expected to collect enough data for evaluation over this period of time. The subjects were required to read the news for about 1 hour per day and provide explicit feedback for each page they visited. ${ }^{1} 28$ users

\footnotetext{
${ }^{1}$ In the last week of the study, some subjects read 2 hours per day. They are encouraged but not required to do so.
}

Figure 2: Web interface after a user logged in.

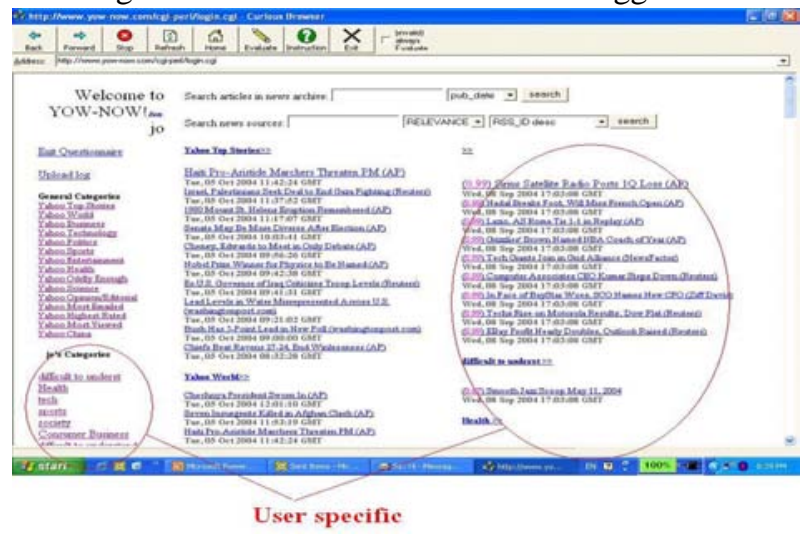

Figure 3: Evaluation user interface. The interface for user to give their explicit feedback of the current news story.

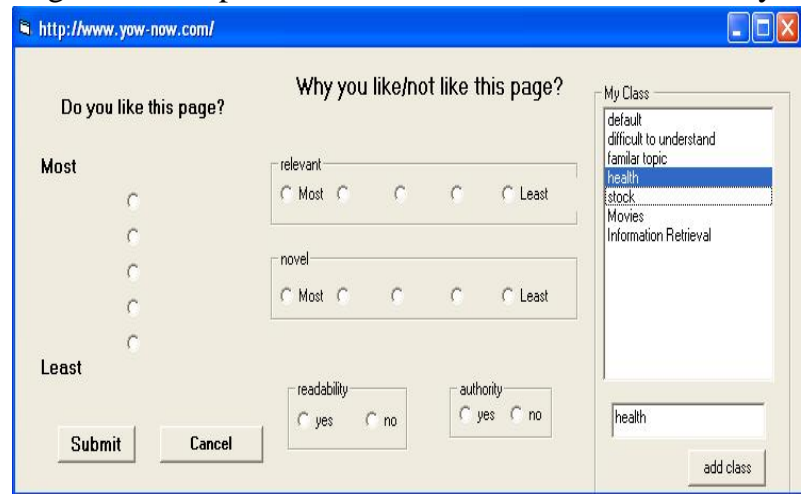

tried this system. However, only 21 users are official paid subjects, among which one worked only for 2 weeks and 20 worked for about 4 weeks.

\subsection{Data collected}

We have collected 7881 feedback entries from all 28 users, among which 7839 were from the 21 official participants. Each entry contains several different forms of evidence for a news story a user clicked. ${ }^{2}$ Our intention to collect the evidence is not to be exhaustive, but representative. The evidence can be roughly classified into the following five categories listed in Tables 1 to $5 .^{3}$

Explicit user feedback After finishing reading a news story, a user clicks a button on the toolbar of the browser to bring up an evaluation interface shown in Figure 3. Through this interface, the user provided the explicit feedback to tell the hidden properties about current story, including the topics the news belongs to (classes), how the user likes this news

\footnotetext{
${ }^{2}$ Each entry is for a $<$ document, user class, time $>$ tuple.

${ }^{3}$ The forms of evidence are listed in the first column and we will get the the other columns later in Section 3 .
} 
(user_likes), how relevant the news is related to the class(es) (relevant), how novel the news is (novel), whether the news matches the readability level of the user (readable), and whether the news is authoritative (authoritative). user_likes, relevant and novel are recorded as integers ranging from 1 (least) to 5 (most). readable and authoritative are recorded as 0 or 1 . A user has the option to provide partial instead of all explicit feedback. A user can create new classes, and choose multiple classes for one documents.

User actions The browser adapted from (Claypool et al., 2001) recorded some user actions, such as mouse activities, scroll bar activities, and keyboard activities (Table 2). TimeOnPage is the number of seconds the user spent on a page, and EventOnScroll is the number of clicks on the scroll bars. When the mouse is out of the browser window or when the browser window is not focused, the browser does not capture any activities. More details about the actions are in (Le and Waseda, 2000).

Topic information Each participant filled out an exit questionnaire and answered several topic/class ${ }^{4}$ specific questions for each of his/her most popular 10 topics and other topics with more than 20 evaluated documents each (Table 3). The questions include how familiar the user is with the topic before the study (topic_familiar_before), how the user likes this topic (topic_like), and how confident the user is with respect to the answers he/she provided (topic_confidence). We include this information as evidence, because they may be collected when a topic is created and used by filtering systems. Whether collecting them in exit questionnaire affects the answers needs further investigation.

News Source Information For each news source (RSS feed), we collected the number of web pages that link to it $\left(R S S_{-}\right.$link), the number of pages that link to the server that provided it (host_link), and the speed of the server that hosts it.

Content based evidence Three pieces of evidence are collected to represent the content of each document: the relevance score, the readability score and the number of words in the document (doc_len) (Table 5). To estimate the relevance score of a document, the system processes all the documents a user put into a class ordered by the feedback time and adaptively learns a topic specific relevance model using the relevance feedback the user provided. The relevance score of a documents is estimated using a

\footnotetext{
4"topic" and "class" are used interchangeably in the paper.
}

Table 1: Basic descriptive statistics about explicit feedbacks.

\begin{tabular}{|c|c|c|c|c|}
\hline Variable & Mean & variance & corr & miss \\
\hline user likes & 3.5 & 1.2 & 1 & 0.05 \\
relevant & 3.5 & 1.3 & 0.73 & 0.005 \\
novel & 3.6 & 1.33 & 0.70 & 0.008 \\
authoritative & 0.88 & 0.32 & 0.50 & 0.065 \\
readable & 0.90 & 0.30 & 0.54 & 0.012 \\
\hline
\end{tabular}

modified logistic regression model learned from all feedback before it (Zhang, 2004). To estimate the readability score of document, the system processes all the documents in all users' classes ordered by the feedback time and adaptively learns a user independent readability model using a logistic regression algorithm.

\section{Preliminary data analysis}

The means and variances of all variables are in Tables 1 to 5. These basic descriptive statistics are very diverse. The values of some evidence may be missing; only the user actions and news source information were always collected. Out of the 7991 entries, only 4522 (57\%) entries contain no missing value. The missing rate of each form of evidence is also reported in the tables. There are several reasons for missing data. For example, the explicit feedback is missing because users didn't always follow instructions, the relevance score is missing for the first story in a class, and the topic_familiar_before values for many topics are missing because we only collected the topic specific answers for larger topics. We expect missing data to be common in operational environments.

The correlation coefficient between each evidence and the explicit feedback user_likes is also listed (corr). The high correlation coefficients between user_likes and other forms of explicit feedback are not very interesting because we can only get explicit feedback after a user reads the document. The correlation coefficient between relevance score and user_likes is 0.37 , the highest among all forms of evidence that the system can get before delivering a document. This is not surprising since most filtering systems only consider relevance and use relevance score to make decisions.

The correlation coefficients between user_likes and the topic information (Table 3) are relatively high. This suggests collecting topic_familiar_before or topic_like in a real filtering system, since they are informative and collecting them requires less user effort (a user only needs to provide information on the class level instead of document level). Section 5 will show how to use it with other forms of evidence in a filtering system. The correlation coefficients between the news source in- 
Table 2: Basic descriptive statistics about user actions. The unit for time is second.

\begin{tabular}{|c|c|c|c|}
\hline Variable & Mean & variance & corr \\
\hline TimeOnPage & $7.2 \times 10^{4}$ & $1.3 \times 10^{5}$ & 0.14 \\
EventOnScroll & 1 & 3.6 & 0.1 \\
ClickOnWindow & 0.93 & 2.5 & 0.05 \\
TimeOnMouse & $2 \times 10^{3}$ & $5.8 \times 10^{3}$ & 0.02 \\
MSecForDownArrow & 211 & 882 & 0.08 \\
NumOfDownArrow & 1.1 & 4.7 & 0.09 \\
MSecForUpArrow & 29 & 240 & 0.03 \\
NumOfUpArrow & 0.10 & 0.8 & 0.04 \\
NumOfPageUp & 0.12 & 0.9 & $\simeq 0$ \\
NumOfPageDown & 0.14 & 1 & $\simeq 0$ \\
MSecForPageUp & 22 & 202 & $\simeq 0$ \\
MSecForPageDown & 28 & 251 & $\simeq 0$ \\
\hline
\end{tabular}

Table 3: Basic descriptive statistics about topics. Each variable ranges from 1 to 7 .

\begin{tabular}{|c|c|c|c|c|}
\hline variable & Mean & variance & corr & miss \\
\hline topic_familiar_before & 3.6 & 1.9 & 0.30 & 0.27 \\
topic_like & 4.9 & 2.0 & 0.30 & 0.27 \\
topic_confidence & 4.7 & 2.0 & 0.34 & 0.27 \\
\hline
\end{tabular}

Table 4: Basic descriptive statistics about news sources.

\begin{tabular}{|c|c|c|c|}
\hline variable & Mean & variance & corr \\
\hline RSS_link & 90.35 & 4.89 & 0.14 \\
host_link & $4.41 \times 10^{4}$ & $7.5 \times 10^{7}$ & 0.08 \\
RSS_SPEED & $3.92 \times 10^{5}$ & $3.7 \times 10^{9}$ & -0.08 \\
\hline
\end{tabular}

Table 5: Basic descriptive statistics about documents. The length of the document does not include HTML tags.

\begin{tabular}{|c|c|c|c|c|}
\hline variable & mean & variance & corr & miss \\
\hline doc_length & 837 & $1.2 \times 10^{3}$ & 0.04 & 0.05 \\
relevant_score & 0.49 & 0.42 & 0.37 & 0.18 \\
readability_score & 0.52 & 0.16 & 0.25 & 0.11 \\
\hline
\end{tabular}

formation and user_likes are weaker (Table 4). The correlation coefficient between user_likes and each user action (Table 2) is even lower (Table 1). Some actions, such as TimeOnPage, are more correlated with user_likes than other refined actions, such as NumOfPageDown. This finding agrees with (Claypool et al., 2001).

\section{Understanding the domain using causal structure learning}

Correlation analysis in Section 3 has helped us to get some initial idea about the data collected. However, in order to better understand the underlying truth of the domain, we need to go beyond correlation and uncover the causal relationships between different variables.

To do that, we first specify $\mathrm{N}$ nodes, one for each form of evidence to be included in the model. Then PC algo- rithm is used (Spirtes et al., 2000) to search the causal relationships between multiple forms of evidence from the data collected. To make the search space smaller, some prior domain knowledge, such as forbidden edges, required edges or temporal tiers, can be introduced before searching. In our experiments, we manually specified some prior knowledge based on the first authors' experience and intuition as the following 5-tier temporal tier: 5 1) Topic info $=$ (familiar_topic_before), $R S S$ info $=\left(\mathrm{RSS} \_\right.$link, host_link $)$, document length (doclen); 2) hidden criteria, such as relevant, novel, authoritative, and readable; 3) system generated scores, such as relevance score and readability score; 4) user_likes; 5) user actions, such as seconds spent on a page (TimeOnPage) or the number of clicks on the $\downarrow$ key (NumOfDownArrow). This informs the learning algorithm that $\rightarrow$ from a higher level to lower level is prohibited.

It is very encouraging to see that the structure learned automatically looks reasonable (Figure 4). According to the graph, novel, relevant, authoritative, readabilty of a document and whether a user is familiar with the topic before using the system (familar_topic_before) are direct causes of the user's preference for a document (user_likes). How familiar with this topic a user is before participating the study (topic_familiar_before) and the number of web links to the news source $\left(R S S_{\text {_link }}\right)$ directly affect the user's relevant and authoritative feedback and readability score. Relevant, authoritative, familiar_topic_before and host_link influence a user's actions, such as the EventOnScroll.

Comparing Tables 2 to 5 with Figure 4, one may ask why some variables are correlated with user likes although there is no direct links between them and user likes. For example, why the correlation between relevance score and user likes is 0.39 , while there is no direct link between them. Does Figure 4 contradict Table 5? The answer is "no". In fact the indirect causal relationship between them tells us why relevance score and user likes are correlated: relevance score and user likes have a common cause relevant. Most of the refined actions, such as the number of pressing page up key (NumOfPageUp), are far away from user_likes. This implies that these refined actions are not very informative if we want to use the learned model to predict whether a user likes a document or not. This finding agree with (Claypool et al., 2001) and Table 2.

The node authoritative is directly linked to readability score and host link. The link between host_link and authoritative confirms the existing approaches that use the web link structure to estimate the

\footnotetext{
${ }^{5}$ Other priors are also possible.
} 
Figure 4: User independent causal graphical structure learned using PC algorithm. $X \rightarrow Y$ means $\mathrm{X}$ is a direct cause of $Y$. $X-Y$ means the algorithm cannot tell if $\mathrm{X}$ causes $\mathrm{Y}$ or if $\mathrm{Y}$ causes $\mathrm{X}$. $X \longleftrightarrow Y$ means the algorithm found some problem, which may happen due to a latent common cause of $\mathrm{X}$ and $\mathrm{Y}$, a chance pattern in the sample, or other violations of assumptions.

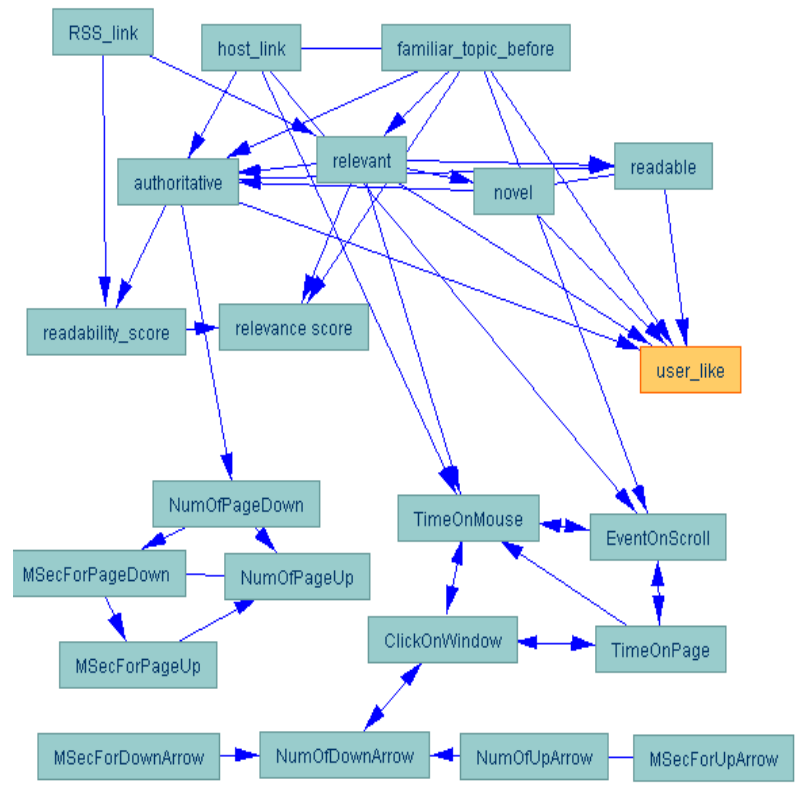

Figure 5: Structure of GM_complete.

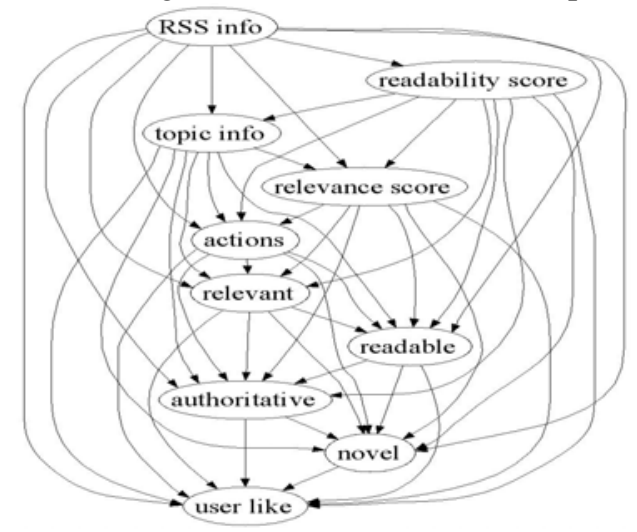

Figure 6: Structure of GM_causal.

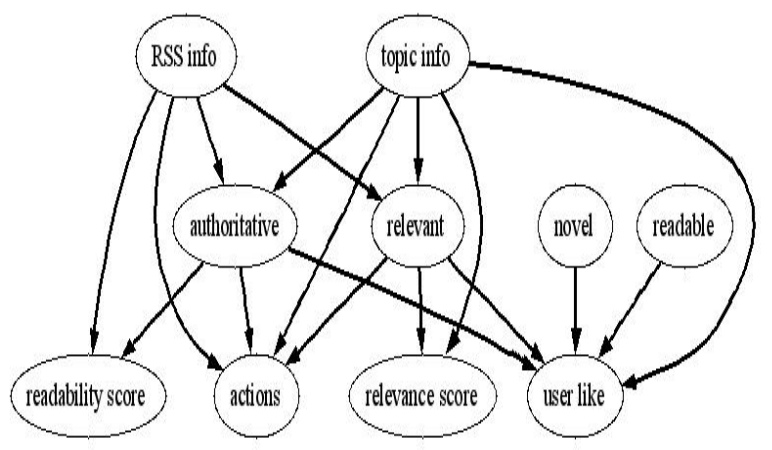

authority of a page (Kleinberg, 1998). The links between readability score, readable and authoritative are very interesting. They suggest the difficulty to understand a page may make the user feel it is not authoritative. Further investigation shows that although the percentage of un-authoritative news is less than $15 \%$ in general, among the 187 news stories some users identified as "difficult" using class labels, $73 \%$ were also rated as not authoritative. Besides some successful web page authority algorithms that only use hyper links, the estimation of authority may be further improved using the content of a page.

There are links among relevant, novel, readable and authoritative. Although the algorithm failed to tell the causal direction between some pairs of variables, it suggests that the four variables influence each other. This may be an inherent property of the document; or because a user is likely to rate one aspect of the document higher than he/she should if the other aspects are good.

One may ask why the structure in Figure 4 contains no link between readable and readability score, since intuitively it should exist. To answer this question, one needs to understand that the causal relationships learned automatically are what the algorithm "believes" based on the evidence of the data, the assumptions it makes, and the prior constraints we engineered. They may have errors, because the data is noisy, or the assumptions and the prior constraints may be wrong. For example, the PC algorithm do statistical test about the independence relationships among variables using the data and the final results are subject to the error of the statistical test. The PC algorithm assumes no hidden variables, however besides relevant, novel, authoritative, and readable, other hidden variables, such as whether a document is up-todate, interesting, misleading, etc. (Schamber and Bateman, 1996), may exist and influence a user's preference for a document. Thus it is not surprising that some of the causal relationships, such as the link between readable and readability score, are missed in the final graph because of the limitation of the learning algorithms. The model learned only sheds some light on the relationships between the variables instead of uncovering the whole truth. It only serves as a starting point for us. To further understand the domain, we may want to break down some variables in the current graph further and relate them to either the user or document properties. In general, causal discovery is inherently difficult and far from solved.

\section{Improving system performance using inference algorithms}

A primary task of a filtering system is to predict user preference (user_likes) for a document so that the system can decide whether to deliver it to the user. To tell whether combining multiple forms of evidence using 
graphical models can improve system performance, we evaluate the proposed solution on the task of predicting user_likes while filtering.

To predict user_likes, the system needs to learn a graphical model: the combination of a graph structure and a set of local conditional probability functions or potential functions. Doing inference over the causal structure learned in the previous section is difficult because of the circles and a mixture of directed and undirected links on the graph. So, we tried the following directed acyclic graphical models.

GM_complete, an almost complete Bayesian network: In this graph, we order the nodes from top to bottom, and the parents of a node are all the nodes above it, such as in Figure 5. For this structure, the order of the nodes is not very important when using Gaussian distributions.

GM_causal, a graphical model inspired by causal models We manually modify the causal structure in Figure 4 to make it a directed acyclic graph as in Figure 6.

In the graphs, RSS info $=\left(R S S \_l i n k\right.$, host_link $)$ and Topic info=topic_familiar_before, topic_like) are 2 dimensional vectors representing the information about the news source and the topic in Table 4 and Table 3. actions $=$ $($ TimeOnPage,...) is a 12 dimensional vector representing the user actions in Table 1. user_likes is the target variable the system wants to predict.

Before learning the parameters of the model, we need to choose a specific conditional form for the probability function associated with each node. We chose Gaussian distributions. If the parents of node $\mathrm{X}$ are $\mathrm{Y}, P(X \mid Y)=$ $N(m+W \times Y, \Sigma)$, where $N(\mu, \Sigma)$ is a gaussian distribution with mean $\mu$ and covariance $\Sigma$. This is a commonly used distribution for continuous valued nodes. It assumes the joint distribution of these variables is multivariate Gaussian, which may be wrong. Nevertheless, because of the mathematical convenience, the existence of efficient learning and inference algorithms for Gaussian networks, and the availability of modeling tools, we chose this distribution. Using the BNT Toolbox (Murphy, 2001), the maximum likelihood estimations of the parameters $(m, W, \Sigma)$ were learned using EM algorithm and junction tree inference engine(Cowell et al., 1999) over the graphical models, with whatever information was available on the first $2 / 3$ of the data.

An alternative approach to combine multiple forms of evidence is linear regression. We tried two special methods to solve the missing evidence problem while using linear regression: 1) building a model that does not use the evidence that is missing for each missing situation (LR_different); or 2)mean substitution: replacing each missing value for an evidence with the average of the

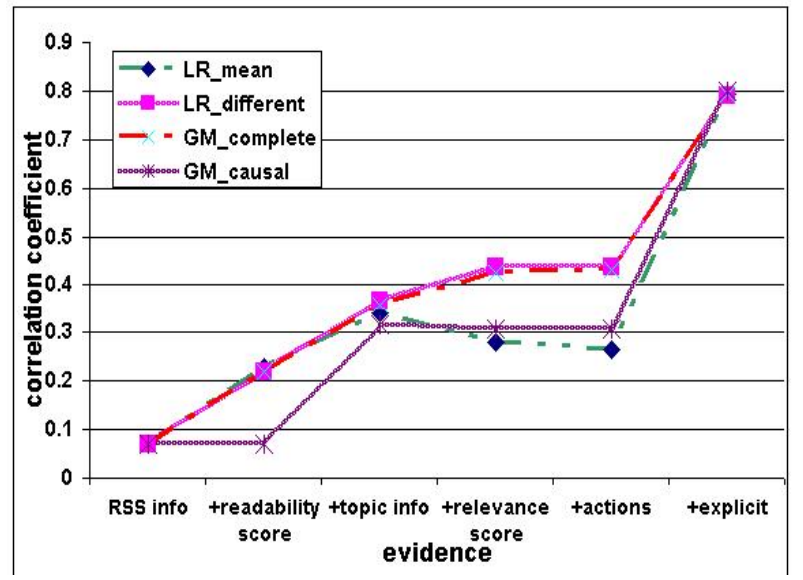

Figure 7: Comparison of the prediction power of different models using 7952 cases for evaluation. The vertical axis is the correlation coefficient between the predicted value of user_likes using the model and the true explicit feedback provided by the users. The order of different forms of evidence is set manually, based on how easy it is to collect each evidence.

observed evidence ( $L R_{-}$mean). For $\mathrm{K}$ different forms of evidence, the system may need to handle $2^{K}$ different evidence missing situations. A large number of linear regression models need to be learned if we use the first approach, considering $\mathrm{K}$ is higher than 15 in some of our experiments. Building $2^{15}$ models is almost impossible for us, so a heuristic approach, which is discussed later, was used to make the experiments possible.

Not all 7991 cases collected in the user study were used in the experiments. We conducted two sets of experiments. For the first set of experiments, we use 7952 cases for which user_likes is not missing. For the other set of runs, we use only cases without missing value. In this task, the value of each variable is continuous and normalized to variance one. Each model is learned using all information available on the first $2 / 3$ of the cases, and tested on the remaining $1 / 3$ of the cases. The correlation coefficient between the predicted value of user_likes and the true explicit user_likes feedback provided by the users is used as the evaluation measure. Our baseline is using relevance score alone, which has a correlation coefficient of 0.367 with $95 \%$ confidence interval $0.33-0.40$ on the last $1 / 3$ of the 7952 cases.

\subsection{Experimental results and discussions}

Figure 7 shows the effectiveness of different models at different testing conditions as indicated by the horizontal axis. From left to right, additional sources of evidence are given when testing. At the very left of the figure $(\mathrm{x}=R S S$ info), a model predicts the 
value of user_likes only given the value of $R S S$ info at testing time. "+explicit" means the explicit feedback (except user_likes) about the current document is given besides the value of actions, relevance score, readability score, $R S S$ info, and TopicInfo. The graphical models and $L R \_$mean model were trained with all evidence/features, and the learned models are independent of the testing condition. LR_different models were only trained with features that are also provided at testing time, so there is one model per testing condition. 6

The results show that GM_complete performs similarly to LR_different. This is not surprising. Theoretically, if there is no missing entries in training data, GM_complete's estimation of the conditional distribution of $P$ (user likes|available evidence) would be the same as that of LR_different on a testing case with missing evidence.

Comparing the correlation coefficients under different testing conditions when using LR_different or GM_complete, we can see that as more forms of evidence are available, the performance improves. If only the news source information of a document ( $R S S$ info) is given, all models perform poorly. The readability score improves the system performance significantly. This is nice and interesting, because the evidence is user independent and can be estimated efficiently for each document. The performance keeps improving as topic info and relevance score were added. To collect them, we needs user feedback on previous documents. The performance improvement is not very obvious with actions added. This means that given other evidence ( $R S S$ info, topic info, relevance score and readability score), the system won't improve its prediction of the document much by observing these actions. However, this is only true when we use a model learned for all users and other forms of evidence are available. It does not mean the actions are useless if we learn user specific model, or if other forms of evidence (such as relevance score) are not available. All models perform very good with explicit feedback added. However, this is a "cheating" condition of less interest to us.

The performances of $L R \_$mean and GM_causal do not increase monotonically as more forms of evidence are added. They perform much worse than LR_different and GM_complete. Why does a structure that looks more causally reasonable not perform well

\footnotetext{
${ }^{6}$ However, for a specific testing condition, the training data and testing data contain cases where some evidence that is supposed to be available is missing. These cases in training data were ignored and not used to learn a $L R_{-}$different model. However, ignoring such kind of cases in testing data makes comparison of different runs difficult. So we used mean substitution approach to fill the required missing features in testing data while using $L R_{-}$different.
}

\begin{tabular}{|c|c|c|c|c|}
\hline Model & Cond. & corr & RLow & RUp \\
\hline LR_mean & $+\mathrm{R}$ & 0.2783 & 0.2426 & 0.3132 \\
LR_different & $+\mathrm{R}$ & 0.4372 & 0.4058 & 0.4677 \\
GM_complete & $+\mathrm{R}$ & 0.4247 & 0.3928 & 0.4555 \\
GM_causal & $+\mathrm{R}$ & 0.3078 & 0.2728 & 0.342 \\
\hline LR_mean & $+\mathrm{A}$ & 0.2646 & 0.2286 & 0.2998 \\
LR_different & $+\mathrm{A}$ & 0.4375 & 0.406 & 0.4679 \\
GM_complete & $+\mathrm{A}$ & 0.4315 & 0.3999 & 0.4622 \\
GM_causal & $+\mathrm{A}$ & 0.3086 & 0.2736 & 0.3428 \\
\hline
\end{tabular}

Table 6: A comparison of different models on all data under the +relevance score $(+\mathrm{R})$ and + action $(+\mathrm{A})$ conditions. Corr is the correlation coefficient between the predicted value of user_likes using the model and the true explicit feedback provided by the users. RLO and RUP are the lower and upper bounds for a $95 \%$ confidence interval for each coefficient.

as the simple GM_complete? We may answer this question better by comparing the underlying assumptions of these algorithms. GM_complete only assumes the joint distribution of all variables is multivariate Gaussian. GM_causal makes much stronger independence assumptions by removing some links between variables. As mentioned before, the causal relationships learned automatically are not perfect, which may cause the poor performance of GM_causal. LR_mean also suffers from the strong conditional independent assumptions.

Table 6 reports the performance together with the confidence intervals of all the models under the +relevance score and +actions conditions. Under both conditions, GM_complete and LR_different are statistically significantly better than the baseline 0.367 . $L R \_m e a n$ and $G M_{\text {_causal }}$ are significantly worse. It means using multiple forms of evidence may hurt some models and benefit others. Further analysis about the + actions runs shows that $L R \_$mean gave explicit feedback too much weight and overlooked other less strong evidence. At testing time, it did not handle the problem of missing explicitfeedback well and thus performed poorly. Although GM_complete also gave very high weights to explicit feedback, it could infer the missing values based on other available evidence at testing time, thus performed better than $L R \_m e a n$. LR_different didn't consider explicit feedback for training, thus it didn't overlook other forms of evidence and suffer from the problem less. $L R \_$mean may work reasonably if explicit variables are not included, however the large difference on how informative each evidence is will still hurt the performance of $L R \_$mean to some extent when some strong evidence is missing. For GM_complete approach, a single model is needed to handle various evidence missing situations. If we use LR_different approach, several models are needed. As we mentioned before, there are $2^{K}$ 


\begin{tabular}{|c|c|c|c|c|}
\hline Model & Cond. & Corr & RLow & RUp \\
\hline LR_mean & $+\mathrm{R}$ & 0.13 & 0.08 & 0.18 \\
LR_different & $+\mathrm{R}$ & 0.41 & 0.37 & 0.45 \\
GM_complete & $+\mathrm{R}$ & 0.41 & 0.37 & 0.45 \\
GM_causal & $+\mathrm{R}$ & 0.41 & 0.375 & 0.45 \\
\hline LR_mean & $+\mathrm{A}$ & 0.11 & 0.061 & 0.16 \\
LR_different & $+\mathrm{A}$ & 0.42 & 0.38 & 0.46 \\
GM_complete & $+\mathrm{A}$ & 0.42 & 0.38 & 0.46 \\
GM_causal & $+\mathrm{A}$ & 0.38 & 0.33 & 0.42 \\
\hline
\end{tabular}

Table 7: The performance on 4522 no missing value cases under the +relevance score $(+\mathrm{R})$ and +action $(+\mathrm{A})$ conditions.

different evidence missing combinations, and $2^{K}$ linear regression models are needed in order to handle all these situations using LR_different approach. LR_different may be preferred if $\mathrm{K}$ is small, while graphical modeling using GM_complete may be a better approach to handle different data missing situations if $\mathrm{K}$ is big.

So far, all results are based on 7952 cases where some evidence may be missing. We also compared the models under different testing conditions using the 4522 cases that do not have any missing value (Table 7). GM_causal performs significantly better than before. We need to be very careful with the structures while using the graphical modeling approach, since a structure that looks more reasonable may work poorly on the inference task. However, we couldn't not draw any conclusion on whether GM_complete is better in general, because the answer may be different with different conditional probability distributions, different data sets, or a better structure learning algorithm.

\section{Related Work}

There has been some research on news filtering using time-coded implicit feedback (Lang, 1995; Morita and Shinoda, 1994). We noticed that an independent work uses a different graphical modeling approach, dependency network, to understand the relationships between implicit measures and explicit satisfaction while user were conducting their web searches and viewing results, and then uses decision tree to predict user satisfaction with results (Fox et al., 2005). Our work differs from the previous work in the goal of the task, the range of evidence considered, the modeling approach we took, and the findings reached.

There has been a lot of related research on using implicit feedback (Kelly and Teevan, 2003). The user actions we collected are based on (Claypool et al., 2001). There is much work about how to handle missing data. (Schafer and Graham, 2002) discussed several approaches such as case deletion, mean substitution, and recommended maximum likelihood (ML) and Bayesian multiple imputation (MI). LR_mean uses mean substitution, LR_different uses case deletion, and graphical models follow the ML approach.

There has been some research on criteria beyond topic relevance (Carbonell and Goldstein, 1998) (Zhang et al., 2002) (Collins-Thompson and Callan, 2004) (Kleinberg, 1998). (Schamber and Bateman, 1996) identified criteria underlying users' relevance judgements and explored how users employed the criteria in making evaluations by asking users to interpret and sort criteria independent of document manually. In the literature, the word "relevant" is used ambiguously, either as a narrow definition of "related to the matter at hand (aboutness)" or a broader definition of "having the ability to satisfy the needs of the user". When it is used by the second definition, such as in (Schamber and Bateman, 1996), researchers are usually studying what we refer to as user likes. In this paper, we use "relevant" as is defined in the first definition and use the phrase "user likes" for the second definition. Despite the vocabulary difference, our work is motivated by the early research. The major contributions of our work in this area are: 1) we model the user likes and other criteria as hidden variables; 2) we quantify the importance of various criteria based on probabilistic reasoning; and 3 ) we have explored the new methodology for combining these criteria with implicit and explicit user feedback.

\section{CONCLUSION}

We have explored how to combine multiple forms of evidence using the graphical modeling approach. This work is significant because it addresses some long-standing issues in the adaptive information filtering community: the integration of a wider range of user-specific and userindependent evidence, and handling situations like missing data that occur in operational environments.

We have analyzed the user study data using graphical models, as well as linear regression algorithms. The experimental results show that the graphical modeling approach can help us to understand the causal relationships between multiple forms of evidence in the domain and explain the real world scenario better. It can also help the filtering system to predict user preference more accurately with multiple forms of evidence compared to using a relevance model only.

As more forms of evidence are added, missing data is a common problem because of system glitches or because users will not behave as desired. A real system needs to handle missing data by either ignoring it or by estimating it based on what is known. The graphical modeling approach addresses this problem naturally. LR_different handles the problem by building many different models to be used at different data missing conditions. LR_different and $G M_{-}$complete perform similarly. When the types 
of evidence is few, LR_different probably is preferable because of the simplicity. However, as more forms of evidence are added, a more powerful model, such as GM_complete, may be preferred because of the computation and space efficiency.

We only collected data for documents users clicked. Further investigation is needed to look at data not clicked, which is a critical step to see whether the improvement on prediction accuracy of user preference will help the system serve the user better in a real system. This is the first step towards using graphical models to combine multiple forms of evidence while filtering. The proposed solution, especially the data analyzing methodology used in this paper, can also be used in other IR tasks besides filtering, such as context-based retrieval.

\section{Acknowledgments}

We thank Jaime Carbonell, Tom Minka, Stephen Robertson, Yiming Yang, Wei Xu, Peter Spirtes, Diane Kelley, Paul Ogilvie, Kevyn Collins-Thompson, Luo Si, Joemon Jose for valuable discussions about the work described in this paper.

This research was funded in part by a fellowship from IBM and a grant from National Science Foundation. Any opinions, findings, conclusions or recommendations expressed in this paper are the authors', and do not necessarily reflect those of the sponsors.

\section{References}

Jaime Carbonell and Jade Goldstein. 1998. The use of MMR, diversity-based reranking for reordering documents and producing summaries. In Proceedings of the 21 st annual international ACM SIGIR conference.

Mark Claypool, Phong Le, Makoto Wased, and David Brown. 2001. Implicit interest indicators. In Intelligent User Interfaces.

K. Collins-Thompson and J. Callan. 2004. A language modeling approach to predicting reading difficulty. In Proceedings of the HLT/NAACL 2004 Conference.

C. Conati, A. S. Gertner, K. VanLehn, and M. J. Druzdzel. 1997. On-line student modeling for coached problem solving using Bayesian networks. In Proceedings of the Sixth International Conference on User Modeling, pages 231-242.

Robert G. Cowell, A. Philip Dawid, Steffen L. Lauritzen, and David J. Spiegelhalter. 1999. Probabilistic Networks and Expert Systems. Springer.

Steve Fox, Kuldeep Karnawat, Mark Mydland, Susan Dumais, and Thomas White. 2005. Evaluating implicit measures to improve web search. In ACM Trans. Information Systems, volume 23.
Donna Harman. 2003. Overview of the TREC 2002 novelty track. In The Eleventh Text REtrieval Conference (TREC-11). NIST 500-251.

E. Horvitz, J. Breese, D. Heckerman, D. Hovel, and K. Rommelse. 1998. The Lumiere project: Bayesian user modeling for inferring the goals and needs of software users. In Proceedings of the Fourteenth Conference on Uncertainty in Artificial Intelligence, July.

Diane Kelly and Jaime Teevan. 2003. Implicit feedback for inferring user preference: a bibliography. SIGIR Forum, 37(2):18-28.

J. Kleinberg. 1998. Authoritative sources in a hyperlinked environment. In Proc. 9th ACM-SIAM Symposium on Discrete Algorithms.

Ken Lang. 1995. Newsweeder: Learning to filter news. In Proceedings of the Twelfth International Conference on Machine Learning.

Phong Le and Makoto Waseda. 2000. A curious browser: Implicit ratings. http://www.cs.wpi.edu/ clay$\mathrm{pool} / \mathrm{mqp} / \mathrm{iii} /$.

Masahiro Morita and Yoichi Shinoda. 1994. Information filtering based on user behavior analysis and best match text retrieval. In Proceedings of the 17th ACM SIGIR conference.

Kevyn Murphy. 2001. The Bayes net toolbox for matlab. In Computing Science and Statistics.

Mark Pilgrim. 2002. What is RSS. http://www.xml.com/pub/a/2002/12/18/dive-intoxml.html.

D.V. Pynadath and W.P. Wellman. 1995. Accounting for context in plan recognition, with application to traffic monitoring. In Proceedings of the Eleventh Conference on Uncertainty in Artificial Intelligence.

Joseph L. Schafer and John W. Graham. 2002. Missing data: Our view of the state of art. In Psychological Methods, volume 7, No 2.

Linda Schamber and Judy Bateman. 1996. User criteria in relevance evaluation: Toward development of a measurement scale. In ASIS 1996 Annual Conference Proceedings, October.

Perter Spirtes, Clark Glymour, and Richard Scheines. 2000. Causation, Prediction, and Search. The MIT Press.

Yi Zhang, Jamie Callan, and Tom Minka. 2002. Novelty and redundancy detection in adaptive filtering. In Proceedings of the 25th Annual International ACM SIGIR Conference.

Yi Zhang. 2004. Using Bayesian priors to combine classifiers for adaptive filtering. In Proceedings of the 27th Annual International ACM SIGIR Conference. 